\title{
Parámetros de crecimiento y sus efectos en la edad al primer empadre de vacas Siboney
}

\section{Growth parameters and their effects on age at first mating of Siboney cows}

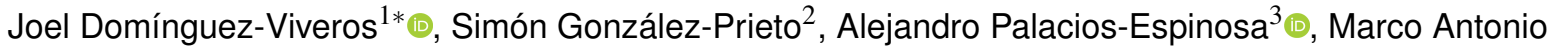

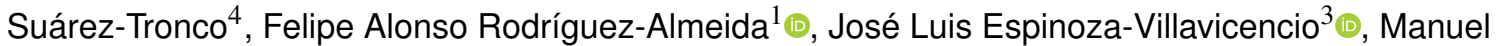 \\ Rodríguez-Castro ${ }^{2}$
}

\footnotetext{
${ }^{1}$ Facultad de Zootecnia y Ecología; Universidad Autónoma de Chihuahua. Periférico Francisco R. Almada Km 1. CP. 31453. Chihuahua, Chihuahua, México.

${ }^{2}$ Centro de Investigaciones para el Mejoramiento Animal de la Ganadería Tropical. Cotorro Ave 101 e/ 100 y 62 No. 6214 Loma de Tierra. La Habana, Cuba.

${ }^{3}$ Departamento de Zootecnia; Universidad Autónoma de Baja California Sur. Carretera al Sur KM 5.5, Apartado Postal 19-B, CP. 23080. La Paz Baja California Sur, México.

${ }^{4}$ Universidad Agraria de la Habana "Fructuoso Rodríguez Pérez". Carretera Tapaste y Autopista Nacional Km $23 \frac{1}{2}$, CP. 32700 , Apartado Postal 18-19. San José de Las Lajas, Mayabeque, Cuba.

*Autor de correspondencia: jodominguez@uach.mx - joeldguezviveros@yahoo.com.mx
}

Nota científica recibida: 20 de marzo de 2018 aceptada: 29 de enero de 2019

RESUMEN. El objetivo fue caracterizar el crecimiento de vacas Siboney con modelos no lineales (MNL) y los efectos de los parámetros de crecimiento sobre la edad al primer empadre (EPE). Se analizaron 10064 datos de peso vivo de 2021 vaquillas. Los MNL evaluados fueron logístico, Gompertz, Brody y Bertalanffy. La selección del mejor modelo fue con el coeficiente de determinación, criterios Akaike y Bayesiano. Con regresión lineal de orden cuadrático, la EPE se analizó con respecto al peso a 24 meses (P24M), tasa de crecimiento (TAC), peso al punto de inflexión (PPI) y madurez a los 24 meses (GM24). El MNL de mejor ajuste fue Bertalanffy; con peso asintótico de $514.3 \mathrm{~kg}$, punto de inflexión a los 5.93 meses con $152.4 \mathrm{~kg}$. Del análisis de regresión: $E P E=(0.026102)(P 24 M)-(0.0000636)\left(P 24 M^{2}\right), E P E=(23.86)(T A C)-(59.17)\left(T^{2}{ }^{2}\right)$, $\mathrm{EPE}=(0.0796)(\mathrm{GM} 24)-(0.000675)\left(\mathrm{GM} 24^{2}\right), \mathrm{EPE}=(0.0299)(\mathrm{PPI})-(0.00009506)\left(\mathrm{PPI}^{2}\right)$, todos diferente de cero $(p<0.01)$. Palabras clave: Bovinos leche, ganadería tropical, modelos no lineales, pubertad, razas sintéticas, tasa de crecimiento.

ABSTRACT. The objectives were to characterize the growth of Siboney cows with nonlinear models (MNL) and the effects of growth parameters on age at first mating (AFM). It was analyzed 10064 live weight data from 2021 heifers. The MNL evaluated were Logistic, Gompertz, Brody and Bertalanffy. The selection of best model was with the coefficient of determination, Akaike and Bayesian criteria. With a linear regression of quadratic order, the AFM was analyzed with respect to the weight at 24 months (W24M), growth rate (GRA), weight at the point of inflection (WPI) and maturity at 24 months (M24M). The MNL of best fit was Bertalanffy; with asymptotic weight of $514.3 \mathrm{~kg}$, inflection point was at 5.93 months with $152.4 \mathrm{~kg}$. From the regression analysis: $A F M=(0.026102)(W 24 M)-(0.0000636)\left(W_{24}{ }^{2}\right), A F M=(23.86)(G R A)-(59.17)\left(G^{2} A^{2}\right), A F M$ $\left.=(0.0796)(\mathrm{M} 24 \mathrm{M})-(0.000675)\left(\mathrm{M} 24 \mathrm{M}^{2}\right), \mathrm{AFM}=(0.0299)(\mathrm{WPI})-(0.00009506)(\mathrm{WPI})^{2}\right)$, all different from zero $(\mathrm{p}<0.01)$.

Key words: Bovine milk, growth rate, non-linear models, puberty, synthetic breeds, tropical cattle ranching.

\section{INTRODUCCIÓN}

Los bovinos Siboney con la composición racial de $62.5 \%$ de Holstein y $37.5 \%$ de Cebú se desarrollaron para las condiciones de pastoreo en las regiones tropicales (López y Ribas 1993, Fernández y Tronco 2011). En la fase productiva, la lactancia se desarrolla a 240 días, se ajusta al modelo de Wood con una curva que incrementa continuamente, con producción máxima de $7.71 \mathrm{~kg}$ a los 40 días en lactancia y $1653 \mathrm{~kg}$ promedio de producción total acumulada (Palacios-Espinosa et al. 2016). La edad al primer parto, y la edad y peso al primer empadre ocurre en promedio a los 35 y 24 meses con peso 
Domínguez-Viveros et al.

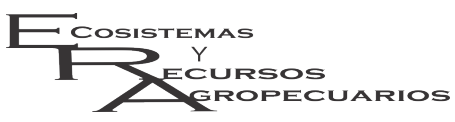

Crecimiento y primer empadre en vacas Siboney

Ecosist. Recur. Agropec.

6(17):383-389,2019

de $306 \mathrm{~kg}$, respectivamente (Portales et al. 2007, Portales et al. 2012). Con relación a las variables de crecimiento, el control de producción contempla el peso vivo en el intervalo del peso al nacer hasta los 950 días de edad (Alonso et al. 2014). Los datos de peso vivo a diferentes edades generan una distribución de puntos a través del tiempo, los cuales permiten describir y analizar el crecimiento animal con base en modelos no lineales (MNL); asimismo, los MNL describen la curva de crecimiento a partir de parámetros con interpretación biológica, como la tasa de crecimiento, el peso adulto o asintótico, grado de madurez, edad y peso al punto de inflexión, entre otros (Domínguez-Viveros et al. 2013, DomínguezViveros et al. 2014).

Las condiciones y los parámetros que definen la curva de crecimiento de las vaquillas tienen repercusiones en la vida productiva y reproductiva de la vaca adulta (Galvão et al. 2009, Roche et al. 2015). Se ha planteado que las vaquillas deben llegar a la primera cubrición con un peso mínimo de 50 a $55 \%$ del peso vivo adulto (Funston y Deutscher 2004, Roberts et al. 2009). El crecimiento de las vaquillas se divide en dos etapas: la prepuberal que se caracteriza por un crecimiento lineal e isométrico, tras la pubertad el crecimiento pasa a ser alométrico, modificándose principalmente la composición corporal y la relación entre los distintos tejidos; y la pubertad o inicio de la etapa reproductiva influyen (Owens et al. 1995, Randel y Welsh 2013). Se sabe que diferencias en las fases del crecimiento pueden presentar consecuencias a lo largo de la vida de la vaquilla, tanto a nivel productivo como reproductivo (Ford y Park 2001, Grings et al. 2007). Con base en lo anterior, el objetivo del presente estudio fue analizar el crecimiento de vacas Siboney con base en MNL; caracterizar la curva de crecimiento a partir de los parámetros de crecimiento derivados de los MNL; y, evaluar los efectos de los parámetros de crecimiento sobre la edad al inicio de la etapa reproductiva.

\section{MATERIALES Y MÉTODOS}

\section{Ajuste y selección de modelos no lineales}

El estudio incluyó 10064 datos de peso vivo, tomados en el intervalo de peso al nacer hasta 950 días de edad, de 2021 vaquillas Siboney nacidas de 2005 a 2015; el número de pesadas por vaquilla osciló de dos a seis, con 4.9 pesadas promedio. Todas las vaquillas se desarrollaron en una empresa pecuaria, ubicada en el municipio Consolación del Sur de la provincia Pinar del Río, República de Cuba. El clima predominante es cálido tropical con dos estaciones, lluviosa de mayo a octubre, y seca de noviembre a abril. La temperatura media anual es de $24{ }^{\circ} \mathrm{C}$, humedad relativa promedio del $80 \%$ con precipitaciones superiores a $3000 \mathrm{~mm}$ anuales. El sistema de alimentación se basó en pastoreo; principalmente, Cynodon nlemfuensis, Digitaria decumbens y Panicum maximum; durante períodos poco lluviosos se suministró suplementación con Saccharum officinarum y Penisetum purpureum (Ribas et al. 2004, Evora et al. 2008)

Se evaluaron cuatro MNL: Logístico (LOG), Gompertz (GOM), Brody (BRO) y von Bertalanffy (BER); los cuales se describen en el Tabla 1, conformados por tres coeficientes de regresión que describen la curva de crecimiento (Agudelo et al. 2008, Domínguez-Viveros et al. 2013). Los análisis se realizaron con el método de Gauss-Newton y el procedimiento NLIN del programa para análisis estadístico SAS (SAS 2005). La selección del modelo con mejor ajuste se realizó en función del error de predicción promedio (EPP), la varianza del error de predicción (VEP), el estadístico de Durbin-Watson (DW), el coeficiente de determinación $\left(R^{2}\right)$, y los criterios de información de Akaike (AIC) y bayesiano (BIC) (Motulsky y Christopoulos 2003, DomínguezViveros et al. 2014, Tiago et al. 2017). Para EPP, VEP, AIC y BIC, el modelo con el menor valor se consideró como el de mejor ajuste; a diferencia del $\mathrm{R}^{2}$, el modelo con el valor más alto representa mejor ajuste. EI DW analiza las auto correlaciones en los errores, con tres planteamientos: si $2<\mathrm{DW} \leq 4$ existe auto correlación negativa; si $0<\mathrm{DW} \leq 2$ revela ausencia de auto correlación; $y$, si $\mathrm{DW}<0$ indica que existe 
Tabla 1. Modelos no lineales evaluados y procedimientos de cálculo de los parámetros de crecimiento.

\begin{tabular}{|c|c|c|c|c|}
\hline Modelo & EcuaciónP & EPI & PPI & GM \\
\hline Brody & $y=\beta 1^{*}\left(1-\beta 2{ }^{*} e^{(-\beta 3 * t)}\right)$ & -- & -- & $\left(1-\beta 2{ }^{*}\left(\exp \left(-\beta 3^{\star} t\right)\right)\right)$ \\
\hline Logístico & $y=\beta 1 /\left(1+\beta 22^{*} e^{(-\beta 3 * t)}\right)$ & $t=\ln \beta 2 / \beta 3$ & $y=\beta 1 / 2$ & $\left.1 /\left(1+\exp \left(-\beta 3^{\star} t\right)\right)\right)$ \\
\hline Bertalanffy & $y=\beta 1^{*}\left(1-\beta 2{ }^{*} e^{(-\beta 3 * t)}\right)^{3}$ & $t=\ln \beta 2 / \beta 3$ & $y=\beta 1 * 8 / 27$ & $\left(1-\beta 2{ }^{*}\left(\exp \left(-\beta 3^{*} t\right)\right)\right)^{3}$ \\
\hline Gompertz & 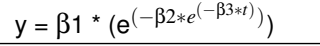 & $t=\ln \beta 2 / \beta 3$ & $y=\beta 1^{*} \exp ^{-1}$ & $\exp \left(-\beta 2^{*}\left(\exp \left(-\beta 3^{*} t\right)\right)\right)$ \\
\hline
\end{tabular}

auto correlación positiva.

\section{Parámetros de crecimiento (PC) y sus efectos en la edad al primer empadre (EPE)}

Con el modelo de mejor ajuste se realizó un análisis de regresión no lineal en las vaquillas con datos de seis pesadas; en 390 vaquillas se logró el criterio de convergencia obteniendo los tres coeficientes de regresión que conforman el MNL, todos estadísticamente diferentes de cero $(p<0.01)$. Posteriormente, para cada vaquilla se estimaron los $P C$ (Tabla 1): a) peso adulto (PAD), a partir del valor asintótico del MNL; b) tasa de crecimiento (TAC), expresando la ganancia de peso como proporción del peso vivo; c) edad y peso (EPI y PPI) al punto de inflexión; d) grado de madurez en porcentaje a los 24 meses de edad (GM24); y, e) peso vivo a los 24 meses de edad (P24M), estimado con base en el MNL. Para analizar los efectos de los PC sobre la edad al primer empadre (EPE; años), se realizaron cuatro análisis de regresión lineal de orden cuadrático con base en los siguientes modelos: $E P E=\beta_{1} \mathrm{P} 24 \mathrm{M}$ $+\beta_{2} \mathrm{P}_{24 \mathrm{M}^{2}}+\varepsilon ;$ EPE $=\beta_{1}$ TAC $+\beta_{2}$ TAC $^{2}+\varepsilon ; E P E=$ $\beta_{1} \mathrm{PPI}+\beta_{2} \mathrm{PPI}^{2}+\varepsilon ; y \mathrm{EPE}=\beta_{1} \mathrm{GM} 24+\beta_{2} \mathrm{GM}_{24}{ }^{2}$ $+\varepsilon$, donde $\beta_{1}$ y $\beta_{2}=$ coeficientes de regresión para el efecto lineal y cuadrático, respectivamente; $\varepsilon=$ residuales. El análisis se realizó con el procedimiento REG de SAS (SAS 2005); para los cuatro modelos de regresión, con base en la primera derivada se obtuvo el punto de inflexión para determinar el punto de cambio en la pendiente de EPE sobre los PC.

\section{RESULTADOS Y DISCUSIÓN}

Con base en la VEP, AIC y BIC el modelo de mejor ajuste para describir el crecimiento fue BER, seguido de GOM, BRO y LOG (Tabla 2). El R ${ }^{2}$, mostró que todos los modelos explican más del 95\% de la variabilidad en los datos. A partir del signo en el EPP los MNL tienden a sobre estimar las predicciones, y el DW exhibió que los residuales no presentan autocorrelaciones. El modelo BER presentó una curva sigmoidea (Figura 1) con PAD de $514.3 \mathrm{~kg}$, el punto de inflexión representó el $29.6 \%$ del PAD, ubicado a los 5.93 meses de edad con 152.4 $\mathrm{kg}$; la correlación negativa y alta entre TAC y PAD (Tabla 2) señala que las vaquillas con peso adulto alto no proceden de altas tasas de crecimiento. El modelo BER se ha reportado como de mejor ajuste para describir el crecimiento de vaquillas Holstein (Berry et al. 2005, Galvão et al. 2009); no obstante, Perotto et al. (1992) y Perotto et al. (1997) reportan como de mejor ajuste el modelo de Richards en el crecimiento de vaquillas Gyr, Guzerat y sus cruzas con Holstein. Las vaquillas en desarrollo representan la nueva generación del avance genético, por lo que es importante su incorporación a la vida reproductiva y productiva de manera idónea. La definición del peso corporal óptimo de las vaquillas en crecimiento depende del sistema de producción y del peso vivo de la vaca adulta a los cuatro años; para un crecimiento optimo, a los 6, 15 y 24 meses de edad la vaquilla debería alcanzar el 30, 60 y $90 \%$ del peso vivo adulto, respectivamente (Ballent et al. 2003, Roche et al. 2015). En vaquillas Siboney, con base en el modelo de BER (Figura 1), el peso vivo a los 6, 15 y 24 meses de edad representó el $16.7,36.1$ y $54.5 \%$ del PAD; el PAD de estos resultados coincide con la clasificación del biotipo liviano de la raza Holstein, y con la clasificación de mayor peso y tamaño de la raza Jersey 
Tabla 2. Resultados para los coeficientes de regresión, parámetros de crecimiento y estadísticos en la selección de los modelos no lineales evaluados.

\begin{tabular}{lllll}
\hline Modelo & Logístico & Brody & von Bertalanffy & Gompertz \\
\hline$\beta 1$ & $356.3 \pm 2.11$ & $10003.7 \pm 6317.6$ & $514.3 \pm 7.35$ & $435.0 \pm 4.38$ \\
$\beta 2$ & $7.788 \pm 0.06$ & $0.997 \pm 0.2$ & $0.602 \pm 0.01$ & $2.529 \pm 0.01$ \\
$\beta 3$ & $0.0047 \pm 0.0001$ & $0.000035 \pm 0.0001$ & $0.0016 \pm 0.0001$ & $0.0024 \pm 0.0001$ \\
$\mathrm{r}_{\beta 1-\beta 3}$ & -0.84 & -0.99 & -0.98 & -0.95 \\
EPI & 7.4 & - & 5.93 & 6.9 \\
$\mathrm{PPI}$ & 178.15 & - & 152.4 & 160.0 \\
$\mathrm{AIC}$ & 64947.4 & 64818.9 & 64652.4 & 64679.4 \\
$\mathrm{BIC}$ & 64969.1 & 64840.5 & 64674.1 & 64701.0 \\
VEP & 634.5 & 626.7 & 616.2 & 617.8 \\
EPP & 5.60 & 2.91 & 4.41 & 5.48 \\
$\mathrm{R}^{2}$ & 0.97 & 0.96 & 0.98 & 0.97 \\
DW & 2.00 & 1.99 & 2.00 & 1.99 \\
\hline
\end{tabular}

Coeficientes de regresión: $\beta 1=$ peso asintótico en $\mathrm{kg} ; \beta 2=$ constante de integración; $\beta 3=$ pendiente de la curva, o tasa de crecimiento en $\mathrm{kg} / \mathrm{kg} / \mathrm{t} ; r_{\beta 1-\beta 3}=$ correlación entre $\beta 1$ y $\beta 3$. Edad (EPI; meses) y peso (PPI; $\mathrm{kg})$ al punto de inflexión. EPP = error de predicción promedio VEP = varianza del error de predicción; $D W=$ estadístico de Durbin-Watson; $R^{2}=$ coeficiente de determinación; $\mathrm{AIC}=$ criterio de información de Akaike; $\mathrm{BIC}=$ criterio de información Bayesiano.

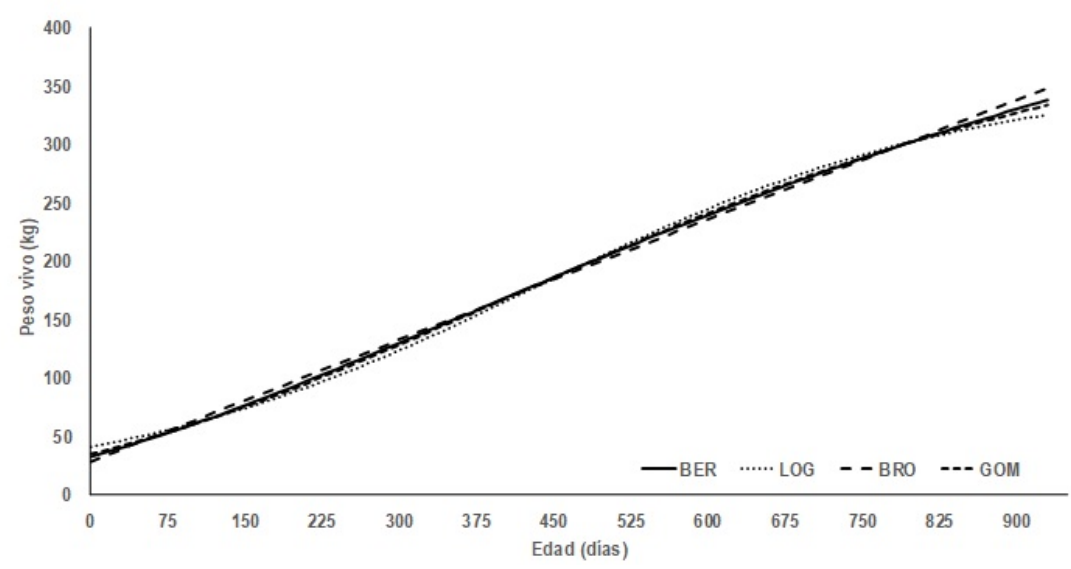

Figura 1. Curvas de crecimiento de vaquillas Siboney con base en los modelos no lineales de von Bertalanffy (BER), logístico (LOG), Brody (BRO) y Gompertz (GOM).

(Ballent et al. 2003, Berry et al. 2005).

La EPE promedio fue de $2.2 \pm 0.26$ años, con promedio de $333.6 \pm 9.52 \mathrm{~kg}$. Los resultados del análisis de regresión fueron: EPE = $(0.026102)(\mathrm{P} 24 \mathrm{M})-(0.0000636)\left(\mathrm{P}_{\left.24 \mathrm{M}^{2}\right)}\right) ; \mathrm{EPE}=$ $(23.86)(T A C)-(59.17)\left(T^{2}{ }^{2}\right) ; E P E=(0.0796)(G M 24)$ - $(0.000675)\left(\mathrm{GM} 24^{2}\right) ; y, \quad \mathrm{EPE}=(0.0299)(\mathrm{PPI})-$ $(0.00009506)\left(\mathrm{PPI}^{2}\right)$; en todos los casos los coeficientes de regresión fueron estadísticamente diferente de cero $(p<0.01)$ y el coeficiente de determinación fue igual o superior a 0.95 . Las cuatro ecuaciones de regresión tuvieron comportamiento similar, al inicio la EPE incrementó junto con la variable de crecimiento evaluada hasta el punto de inflexión o cambio del signo en la pendiente, con valores de $205.2 \mathrm{~kg}$ en P24M, $0.2016 \%$ en TAC, 58.9\% en GM24 y $157.3 \mathrm{~kg}$ en PPI; a partir del punto de inflexión por cada unidad de incremento en los PC se observaron efectos para reducir EPE; en la Figura 2 se presenta el comportamiento de la EPE a través del P24M.

La edad puede ser un modulador significativo del inicio de la pubertad, siempre y cuando los animales estén en un plano nutricional adecuado en las primeras fases del desarrollo. El inicio de la fase reproductiva es un proceso presidido por una señal nutricional, el cerebro requiere reconocer cierto grado de desarrollo corporal y madurez para iniciar las funciones del mecanismo fisiológico que controlan 


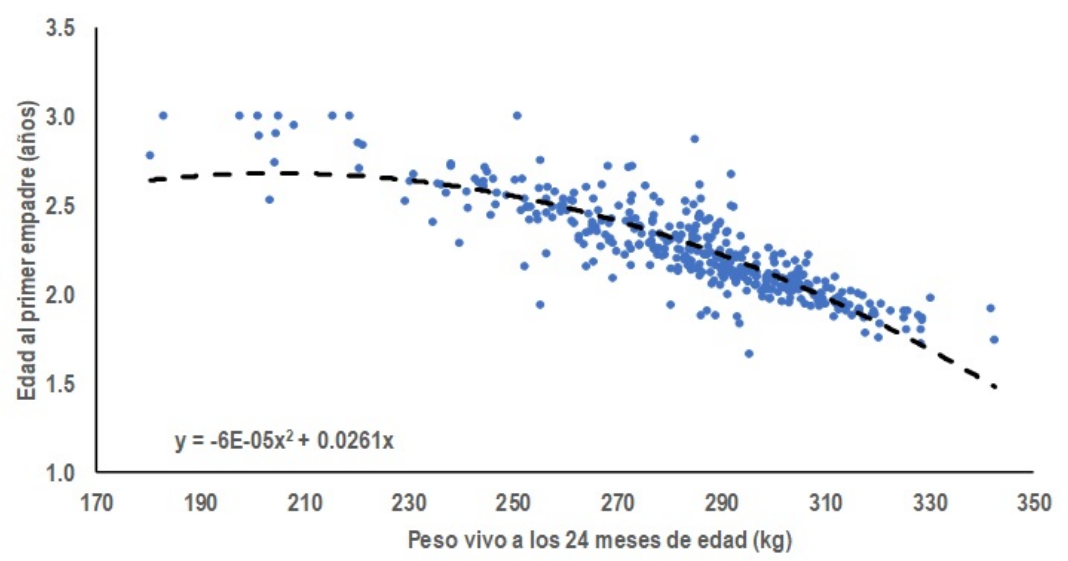

Figura 2. Relación de la edad al primer empadre y el peso vivo a los 24 meses de edad, producto del análisis de regresión lineal de orden cuadrático.

la reproducción, lo cual sustenta el criterio de que el tamaño corporal es más importante que la tasa de crecimiento, dado que el crecimiento y conformación del aparato reproductor constituyen un proceso gradual que requiere de cierto grado de madurez del animal (Placen et al. 1998, Foster y Nagatani et al. 1999). Otros autores han propuesto que la pubertad ocurre en el punto de inflexión de la curva de crecimiento, en ese punto se realiza un cambio en la tasa de crecimiento y en la tasa de deposición de tejido magro a tejido graso, asociando el retraso de la pubertad a una menor deposición de grasa (Randel y Welsh 2013); el tejido adiposo desempeña un papel importante en los mecanismos que regulan los factores de crecimiento y las hormonas reproductivas (Zieba et al. 2005, Hausman et al. 2012). Las vaquillas pueden alcanzar la pubertad a un peso similar, pero no ocurre con una composición corporal o estado metabólico y endocrino similar (Hall et al. 1995).

Con el manejo y alimentación de la vaquilla en crecimiento se debe planear un peso y desarrollo corporal adecuado para el primer parto, con la intensión de evitar repercusiones en el rendimiento por lactancia y la longevidad o vida productiva de la vaca. El modelo de von Bertalanffy fue el de mejor ajuste para describir el crecimiento de las vaquillas Siboney, presentó una curva sigmoidea con punto de inflexión a los 5.93 meses de edad con $152.4 \mathrm{~kg}$. Los parámetros de crecimiento mostraron efecto sobre la edad al primer empadre y al inicio una pendiente positiva, a partir de este punto de cambio se observaron efectos para reducir la edad de inicio de la etapa reproductiva.

\section{LITERATURA CITADA}

Agudelo GDA, Cerón MM, Restrepo BL (2008) Modelación de las funciones de crecimiento aplicadas a la producción animal. Revista Colombiana de Ciencias Pecuarias 21: 39-58.

Alonso AC, Chongo B, Zamora A, Torres V, Noda AC, Acosta F (2014) Curvas de crecimiento de hembras en desarrollo Siboney de Cuba, hasta 18 meses de edad. Revista Cubana de Ciencia Agrícola 48: 227-233.

Ballent M, Landi HG, Bilbao G, Dick A (2003) Pubertad, pero vivo y desarrollo corporal en diferentes biotipos bovinos productores de leche: una actualización bibliográfica. Información Técnica Económica Agraria 2: 130-138.

Berry DP, Horan B, Dillon P (2005) Comparison of growth curves of three strains of female dairy cattle. Animal Science 80: 151-160. 
Domínguez-Viveros J, Rodríguez-Almeida FA, Núñez-Domínguez R, Ramírez-Valverde R, Ortega-Gutiérrez JA, Ruiz-Flores A (2013) Ajuste de modelos no lineales y estimación de parámetros de crecimiento en bovinos Tropicarne. Agrociencia 47: 25-34.

Domínguez-Viveros J, Ortega-Gutiérrez JA, Rodríguez-Almeida FA, Callejas-Juárez N, Aguilar-Palma NG, Santillán-Moreno E (2014) Ajuste de modelos no lineales para caracterizar el crecimiento de bovinos Hereford y Salers. Revista Científica FCV-LUZ 24: 436-442.

Evora JC, Portales A, González-Peña D, Guerra D (2008) Relación entre las épocas de incorporación y parto y la eficiencia productiva de hembras Siboney de Cuba primíparas. Ciencia y Tecnología Ganadera 2: 97-101.

Fernández FJ, Tronco MA (2011) Influencia de factores no genéticos en la producción de leche del Siboney de Cuba. Revista Salud Animal 33: 76-82.

Ford JA, Park CS (2001) Nutritionally directed compensatory growth enhances heifer development and lactation potential. Journal of Dairy Science 84: 1669-1678.

Foster DL, Nagatani S (1999) Physiological perspectives on leptin as a regulator of reproduction: role in timing puberty. Biology of Reproduction 60: 205-215.

Funston RN, Deutscher GH (2004) Comparison of target breeding weight and breeding date for replacement beef heifers and effects on subsequent reproduction and calf performance. Journal of Animal Science 82: 3094-3099.

Galvão CJ, Franklin BP, Tonhati H, Ramalho de FM (2009) Análise das relações da curva de crescimiento e eficiência productiva de vacas raça holandesa. Revista Brasileira de Zootecnia 38: 2346-2353.

Grings EE, Geary TW, Short RE, MacNeil MD (2007) Beef heifer development within three calving systems. Journal of Animal Science 85: 2048-2058.

Hall JB, Staigmiller RB, Bellows RA, Short RE, Moseley WM, Bellows SE (1995) Body composition and metabolic profiles associated with puberty in beef heifers. Journal of Animal Science 73: 3409-3420.

Hausman G, Barb C, Lents C (2012) Leptin and reproductive function. Biochimie 94: 2075-2081.

López D, Ribas M (1993) New dairy cattle breeds. Results in Cuba. Revista Cubana de Ciencia Agrícola 27: $1-10$.

Motulsky H, Christopoulos A (2003) Fitting models to biological data using linear and nonlinear regression. A practical guide to curve fitting. Graph Pad Software Inc. USA. 351p.

Owens FN, Gill DR, Secrist DS, Coleman SW (1995) Review of some aspects of growth and development of feedlot cattle. Journal of Animal Science 73: 3152-3172.

Palacios-Espinosa A, Domínguez-Viveros J, Padrón-Quintero Y, Rodríguez-Castro M, Rodríguez-Almeida FA, Espinoza-Villavicencio JL, et al. (2016) Caracterización de la curva de lactancia de bovinos siboney con modelos no lineales mixtos. Revista Mexicana de Ciencias Pecuarias 7: 233-243.

Perotto D, Cue RI, Lee AJ (1992) Comparison of nonlinear functions for describing the growth curve of three genotypes of dairy cattle. Canadian Journal of Animal Science 72: 773-782.

Perotto D, de Paula CMJ, da Rocha JL, Martins PJ (1997) Descrção das curvas de crescimento de fêmaeas bovinas Guzerá, Gir, Holandês $x$ Guzerá e Holandês $x$ Gir. Revista Brasileira de Zootecnia 26: 283-288.

Placen NT, Heinrichs AJ, Erb HN (1998) The effects of disease, management, and nutrition on average daily gain of dairy heifers from birth to four months. Journal of Dairy Science 81: 1004-1009. 
Portales A, González-Peña D, Guerra D, Ortiz J (2007) Parámetros genéticos para rasgos reproductivos de primer parto en el genotipo Siboney de Cuba. Ciencia y Tecnología Ganadera 1: 143-148.

Portales A, González-Peña D, Guerra D, Évora JC, Acosta M (2012) Parámetros genéticos de las características de leche, incorporación y parto en ganado Siboney de Cuba. Ciencia y Tecnología Ganadera 6: 27-33.

Randel RD, Welsh TH (2013) Interactions of feed efficiency with beef heifer reproductive development. Journal of Animal Science 91: 1323-1328.

Ribas M, Gutiérrez M, Mora M, Evora JC, González S (2004) Comportamiento productivo y reproductivo del Siboney de Cuba en dos localidades. Revista Cubana de Ciencia Agrícola 38: 121-126.

Roberts AJ, Geary TW, Grings EE, Waterman RC, MacNeil MD (2009) Reproductive performance of heifers offered ad libitum or restricted access to feed for a one hundred forty-day period after weaning. Journal of Animal Science 87: 3043-3052.

Roche JR, Dennis NA, MacDonald KA, Phyn CV, Amer PR, White RR, et al. (2015) Growth targets and rearing strategies for replacement heifers in pasture-based systems: a review. Animal Production Science 55: 902-915.

SAS (2005). User's Guide: Statistics. Version 9.0. Statistical Analysis System. SAS Institute Inc. Cary NC, USA. 5121.

Tiago TJ, Cazonatto GA, da Silva RW (2017) Comparing non-linear mathematical models to describe growth of different animals. Acta Scientiarum Animal Sciences 39: 73-81.

Zieba DA, Amstalden M, Williams GL (2005) Regulatory roles of leptin in reproduction and metabolism: A comparative review. Domestic Animal Endocrinology 29: 166-185. 
